\title{
Visualizing the collapse and revival of wavepackets in the infinite square well using expectation values
}

\author{
R. W. Robinett* \\ Department of Physics \\ The Pennsylvania State University \\ University Park, PA 16802 USA
}

(Dated: November 3, 2018)

\begin{abstract}
We investigate the short-, medium-, and long-term time dependence of wave packets in the infinite square well. In addition to emphasizing the appearance of wave packet revivals, i.e., situations where a spreading wave packet reforms with close to its initial shape and width, we also examine in detail the approach to the collapsed phase where the position-space probability density is almost uniformly spread over the well. We focus on visualizing these phenomena in both position- and momentum-space as well as by following the time-dependent expectation values of and uncertainties in position and momentum. We discuss the time scales for wave packet collapse, using both an autocorrelation function analysis, as well as focusing on expectation values and find two relevant time scales which describe different aspects of the decay phase. In an Appendix, we briefly discuss wave packet revival and collapse in a more general, one-dimensional power-law potential given by $V_{(k)}(x)=V_{0}|x / a|^{k}$ which interpolates between the case of the harmonic oscillator $(k=2)$ and the infinite well $(k=\infty)$.
\end{abstract}

*Electronic address: rick@phys.psu.edu 


\section{Introduction}

The study of wave packet solutions of the Schrödinger equation in model potentials can illuminate many aspects of wave mechanics, both semi-classical features which have an obvious classical analog, as well as purely quantum mechanical effects. Since the first numerical studies of wave packet motion 1] appeared in this journal, there have been a large number of papers illustrating such phenomena as wave packet interactions with square barriers or wells [2], multi-well systems [3], multi-dimensional scattering [4], and in systems of relevance to solid-state physics [5]. Popular simulation packages [] now allow students to easily visualize the time-evolution of quantum states in a number of systems (as opposed to the more traditional static images of single stationary state solutions) and allow them to systematically vary the values of important physical parameters to study the dependence on such quantities as the particle mass, incident energy, and barrier/well properties such as height/depth and width.

Such studies have become increasingly relevant as pedagogical tools as wave packet propagation in more realistic quantum mechanical bound state systems has been probed experimentally, especially the behavior of Coulomb wave packets on circular 7] or elliptical 8] orbits which are accessible in Rydberg atom states [9]. One of the most intriguing aspects of such systems is that initially localized wave packets, which have a short-term time evolution which exhibits simple classical behavior, will spread significantly after several orbits, entering a so-called collapsed phase, only to reform later in the form of a quantum revival in which the spreading reverses itself and the wave packet relocalizes.

One of the early papers to point out the possibility of such collapse/revival behavior in the context of the infinite well was by Segre and Sullivan [10]. They studied the motion of bound state wave packets in such a system using a simple "sum over energy eigenstates" method. These authors noted, in passing only, many of the same aspects observed in the Coulomb system, namely the spatial spreading of the wave packet, continuing until the

probability density is almost uniformly spread over the entire well, and then reforming into something like the $t=0$ configuration.

More recently, Bluhm, Kostelecký, and Porter [11] have examined the time evolution and revival structure of wave packets in more generic one-dimensional systems (examining the harmonic oscillator and infinite well in particular as special cases) while Aronstein and 
Stroud [12] have focused on fractional revivals [13] in the infinite well, also presenting visualizations of position-space probability densities. These studies have made extensive use of the autocorrelation function introduced by Nauenberg [14], defined by

$$
\begin{aligned}
C(t) & =\int_{-\infty}^{+\infty} \psi^{*}(x, t) \psi(x, 0) d x \\
& =\int_{-\infty}^{+\infty} \phi^{*}(p, t) \phi(p, 0) d p \\
& =\sum_{n=0}^{\infty}\left|a_{n}\right|^{2} e^{i E_{n} t / \hbar}
\end{aligned}
$$

where the final form is useful if the bound state wave packet is written in terms of energy eigenstates, $u_{n}(x)$, via

$$
\psi(x, t)=\sum_{n=0}^{\infty} a_{n} u_{n}(x) e^{-i E_{n} t / \hbar}
$$

The quantity $C(t)$, which measures the overlap of the initial state wave packet (in either position- or momentum-space) with the state at later times, can be used in its last form to derive information about the classical period of motion, as well as revival and superrevival times [11], using information on the $n$-dependence of the $E_{n}$.

While the autocorrelation function is a powerful tool for such formal and general analyses 15], plots of the time-dependence of $C(t)$ are rather distant from the intuitive picture of initially semi-classical particle motion in the well, accompanied by wave packet spreading, leading to a collapsed state. Plots of position-space probability densities, such as those in Ref. [12] for isolated times, contour plots $\left(|\psi(x, t)|^{2}\right.$ versus $\left.(x, t)\right)$ for all $t$ up to a revival period (as in the 'quantum carpet' visualization in Ref. 16]), or even in the form of more dynamic animations, all can give valuable information on the behavior of the quantum state in position-space as a function of time.

A much more direct connection, however, to an intuitive classical description involving particle trajectories can be obtained by evaluating the time-dependent expectation values of standard variables such as position and momentum, $\langle x\rangle_{t},\langle p\rangle_{t}$, which can then be compared to familiar classical results, illustrating the expected quasi-classical behavior over the first few periods, but also demonstrating the results of purely quantum effects during the collapse and various revival phases. Since the collapse is due to the spreading of the wave packet, it is also natural to 'track' the behavior of the spreads or uncertainties in the same variables, $\Delta x_{t}, \Delta p_{t}$, as functions of time, examining as well how these behave during the semi-classical, 
collapse, and revival phases. Taken together, these can offer a natural visualization and complementary description of the quantum state over all of the natural time scales in the problem, including the semi-classical and collapsed phases which have not been examined in as much detail as the important revival structures. While the results we present here will be evaluated numerically, our approach is the same as that taken by Styer [17] in understanding wave packet propagation through expectation values and uncertainties.

In this note, we begin by very briefly reviewing the autocorrelation approach to the revival structure of wave packet propagation in the infinite well in Section IIA. We illustrate this with calculations, not only of the standard $C(t)$ over the revival period, but of a related correlation function which provides information on the reformation of the wave packet at half-revival periods as well as providing additional illustrative evidence for fractional revivals. Probability density plots, in both position- and momentum-space, are also provided to illustrate the connections to the autocorrelation function approach as we review and extend the results in Refs. [11] and [12], but also for later comparison to results obtained from expectation values. In Section IIB we focus on the short-term evolution of wave packets, visualizing through expectation values and uncertainties in both $x$ and $p$, the quasi-classical propagation and the quantum spreading leading to collapse. In Section IIC, we repeat this analysis, but instead focus on the entire revival period, providing more graphic evidence of the reformation at the half-revival time. In contrast to earlier works, in Section IID, we focus on the medium-term time dependence, emphasizing the approach to the collapsed phase. We study the decay in the degree of correlation between the initial packet and later states (as measured by $|C(t)|$ ) as well as the approach to the quasi-uniform or 'flat' probability density phase discussed by Segre and Sullivan [10]. We find that the time scale for the initial loss of coherence as measured by $|C(t)|$ is simply related to the free particle spreading time, $t_{0}$, while that which describes the approach to the 'flat' or truly collapsed state, $t_{\text {flat }}$ (as given by the approach of $\langle x\rangle_{t},\langle p\rangle_{t}$, and most obviously $\Delta x_{t}$ to their 'flat' values), are, in fact, different and we examine both in detail. In this regard, we find that an approach involving expectation values and uncertainties provides useful information which is not encoded so obviously in the autocorrelation approach.

Finally, in an Appendix, we consider wave packet revivals in a more general, onedimensional power-law potential given by $V_{(k)}(x)=V_{0}|x / a|^{k}$ as this form interpolates smoothly between the case of the harmonic oscillator $(k=2)$ and the infinite well $(k=\infty)$. 
Using simple WKB methods for the estimation of the energy eigenvalues (as was done in Ref. [18]) and the methods in Ref. [11], we can explicitly exhibit the scaling of the revival time with $k$ which gives the familiar result for the infinite well and which also exhibits the appropriate divergence $\left(T_{\text {rev }} \rightarrow \infty\right.$ as $\left.k \rightarrow 2\right)$ as one approaches the harmonic oscillator case. For this same general system, we also evaluate the revival times for half-wells where one introduces an infinite wall at the origin and restricts the particle in $V_{(k)}(x)$ to have $x>0$. Gea-Banacloche [19] has recently examined the case of a 'quantum bouncing ball' in such a potential $(V(z)=m g z$ for $z>0$ so that $k=1)$ and we find that our general result reproduces that special case.

\section{Visualizing wavepacket revivals and collapse}

While the revival structure of wave packets in the infinite well has been discussed in detail in Refs. [11] and [12], we will briefly recall the most important points of an autocorrelation function analysis of this system, extending these results slightly and providing some complementary visualizations of related topics.

\section{A. Autocorrelation function analysis}

For definiteness, we consider the one-dimensional infinite square well system, with walls at $x=0, L$, for which the most general time-dependent solution of the Schrödinger equation (since this system does not allow for unbound or continuum states) is given by the infinite discrete summation

$$
\psi(x, t)=\sum_{n=1}^{\infty} a_{n} u_{n}(x) e^{-i E_{n} t / \hbar}
$$

where the (normalized) eigenstates and energy eigenvalues are, of course,

$$
u_{n}(x)=\sqrt{\frac{2}{L}} \sin \left(\frac{n \pi x}{L}\right) \quad \text { and } \quad E_{n}=n^{2} \frac{\hbar^{2} \pi^{2}}{2 m L^{2}}
$$

Recall that the normalization of the position- and momentum-space wavefunctions are related via

$$
1=\sum_{n=1}^{\infty}\left|a_{n}\right|^{2}=\int_{0}^{L}|\psi(x, t)|^{2} d x=\int_{-\infty}^{+\infty}|\phi(p, t)|^{2} d p=1
$$


Because we are interested in evaluating expectation values of many quantities for somewhat detailed comparison, we will be careful to ensure that any wave packet is appropriately normalized.

Because the quantized energy eigenvalues are integral multiples of a common value, it is easy to see that any such solution will exhibit revivals or reformations, where $\psi(x, t+T)=$ $\psi(x, t)$, when

$$
e^{-i E_{n} T / \hbar}=+1 \quad \text { or } \quad T=\frac{4 m L^{2}}{\hbar \pi}
$$

which will be called the revival time. As shown in Refs. [12] and [16], the wave packet will also reform itself at half this time as well, but in a possibly different location. We see this by noting that

$$
\begin{aligned}
\psi(L-x, t+T / 2) & =\sum_{n=1}^{\infty} a_{n} u_{n}(L-x) e^{-i E_{n}(t+T / 2) / \hbar} \\
& =\sum_{n=1}^{\infty} a_{n} u_{n}(x) e^{-i E_{n} t / \hbar}\left[-\cos (n \pi) \cos \left(n^{2} \pi\right)\right] \\
& =-\psi(x, t)
\end{aligned}
$$

This connection can be written in the form

$$
\psi(L-x, t+T / 2)=-\psi(x, t) \quad \Longrightarrow \quad \psi(x, t+T / 2)=-\psi(L-x, t)
$$

or

$$
|\psi(x, t+T / 2)|^{2}=|\psi(L-x, t)|^{2}
$$

so that at half the revival time later, any initial wavepacket will reform itself (same shape, width, etc.), but at a location mirrored about the center of the well.

The momentum behavior of the system will be given by

$$
\phi(p, t)=\frac{1}{\sqrt{2 \pi \hbar}} \int_{0}^{L} \psi(x, t) e^{i p x / \hbar} d x
$$

at all times and we can use this connection to understand the behavior of the wave packet in momentum space at $T / 2$. We note that

$$
\begin{aligned}
\phi(p, t+T / 2) & =\frac{1}{\sqrt{2 \pi \hbar}} \int_{0}^{L} \psi(x, t+T / 2) e^{i p x / \hbar} d x \\
& =-\frac{1}{\sqrt{2 \pi \hbar}} \int_{0}^{L} \psi(L-x, t) e^{i p x / \hbar} d x \\
& =-e^{i p L / \hbar}\left[\frac{1}{\sqrt{2 \pi \hbar}} \int_{0}^{L} \psi(y, t) e^{-i p y / \hbar} d y\right] \\
& =-e^{i p L / \hbar} \phi(-p, t)
\end{aligned}
$$


so that

$$
|\phi(p, t+T / 2)|^{2}=|\phi(-p, t)|^{2}
$$

and half a revival time later the initial momentum profile is also reproduced, except flipped in sign $(p \rightarrow-p)$, so that the particle is moving in the 'other direction'. These statements are true of any general time-dependent solution of the form in Eqn. (3) for the infinite square well.

For a typical wave packet solution, the expansion coefficients are such that they are sharply peaked about some large value of $n$, say $n_{0}>>1$, so that the packet can be characterized by a momentum value $p_{0} \sim n_{0} \pi \hbar / L$. The period of the classical motion will be given by $\tau=2 L / v_{0}$ and if we associate $v_{0}=p_{0} / m$ we find that

$$
\tau=\frac{2 L}{p_{0} / m}=\frac{2 L^{2} m}{n_{0} \pi \hbar}=\frac{1}{2 n_{0}}\left[\frac{4 m L^{2}}{\hbar \pi}\right]=\frac{1}{2 n_{0}} T
$$

(See also Ref. [11] for a more general discussion of the classical period.) We thus expect the wave packet to undergo many classical periods before reforming itself in a revival and note that there are at least two important time scales in the problem, namely $T>>$.

As an explicit example of a bound-state wave packet, we will use a quasi-Gaussian form, with expansion coefficients given by

$$
a_{n}=\sqrt{\frac{\alpha \hbar \sqrt{\pi}}{L}} e^{-\alpha^{2}\left(p_{n}-p_{0}\right)^{2} / 2} e^{-i p_{n} x_{0} / \hbar}
$$

where

$$
p_{n} \equiv \frac{n \pi \hbar}{L} \quad \text { and } \quad p_{0} \equiv \frac{n_{0} \pi \hbar}{L}
$$

define the central momentum, corresponding to an initial speed to the right of $v_{0} \sim p_{0} / m$. The initial position of the wave packet is then given by $x_{0}$. (This is similar to the form used in Ref. [10].)

This form gives an initial wave packet which is almost Gaussian and hence very similar in most regards to the standard analytic result seen in many textbooks where explicit freeparticle wave packets are constructed. (See, for example, Refs. [20], 21].) For example, with this normalization, the expansion coefficients are already almost normalized (but we explicitly ensure the proper normalization in all of our numerical calculations) and the initial uncertainties or spreads in the wave packet are given (to an excellent approximation) by

$$
\Delta p=\frac{1}{\alpha \sqrt{2}} \quad \text { and } \quad \Delta x_{0}=\frac{\alpha \hbar}{\sqrt{2}}
$$


provided $\Delta x_{0}<<L$. We recall that the corresponding free particle wave packet with Gaussian shape given by the continuous momentum weighting

$$
\phi(p)=\sqrt{\frac{\alpha}{\sqrt{\pi}}} e^{-\alpha^{2}\left(p-p_{0}\right)^{2} / 2} e^{-i p x_{0} / \hbar}
$$

spreads with time with a spatial uncertainty given analytically by

$$
\Delta x_{t}=\Delta x_{0} \sqrt{1+\left(\frac{t}{t_{0}}\right)^{2}} \quad \text { where } \quad t_{0} \equiv m \hbar \alpha^{2}=\frac{2 m\left(\Delta x_{0}\right)^{2}}{\hbar}
$$

This spreading time, $t_{0}$, defines a third time scale in the problem which we can write in the following form

$$
\frac{t_{0}}{\tau}=n_{0} \pi\left(\frac{\Delta x_{0}}{L}\right)^{2}
$$

for future comparison. For our explicit calculations, we choose the numerical values $2 m=$ $\hbar=L=1$ to define the model system and for the specific wave packet solution we use

$$
\Delta x_{0}=0.05 \quad \longrightarrow \quad \alpha=\frac{1}{10 \sqrt{2}} \quad \longrightarrow \quad \Delta p=10
$$

with

$$
x_{0}=0.5 \quad \text { and } \quad n_{0}=400
$$

With these values we have $T / \tau=2 n_{0}=800$ and $t_{0} / \tau=\pi$ so that the wave packet will spread significantly on the time scale of a few classical periods.

Using this explicit wave packet, we can evaluate the autocorrelation function given by Eqn. (11) and we plot $|C(t)|$ versus $t$ over half a revival period in Fig. 1. (The plot is symmetric about the half-revival time, T/2.) The initial decrease in correlation for $t \gtrsim 0$ is seen as the 'decay' in $|C(t)|$, while the structures in $C(t)$ due to many of the fractional revivals [12], 13] are also apparent. In order to show evidence for the reformation at $T / 2$ mentioned above, we also show in Fig. 1 a plot of a related correlation function defined by

$$
\begin{aligned}
\bar{C}(t) & \equiv \int_{-\infty}^{+\infty} \psi^{*}(L-x, t) \psi(x, 0) d x \\
& =\left[\int_{-\infty}^{+\infty} \phi^{*}(-p, t) \phi(p, 0) d p\right] e^{+i p L / \hbar}
\end{aligned}
$$

which measures the correlation between the initial wave packet and later states which are 'flipped' about the center of the well or, equivalently, reappear with opposite momentum profiles. Using this form, the reformation at $T / 2$ is clearly seen $(|\bar{C}(T / 2)|=1)$ as well as additional evidence for fractional revivals. 
Also using this specific wave packet as an example, we illustrate the position-space probability densities at several times in Fig. 2. The initial, centered wave packet at $t=0$ (solid curve) can be seen later at several obvious fractional revival times. The packet at $t=\tau / 8$ (dashed curve) can also be seen one-half revival time later, reformed on the other side of the well as dictated by Eqn. (91). The position-space probability density at a more 'random' later time $(t=124 \tau)$ illustrates the observation of Ref. [10] where the position-space probability has become almost equally distributed over the entire well; the arrow indicates the magnitude of a truly uniform probability distribution given by $P_{C L}(x)=1 / L$ (corresponding to the numerical values used here.)

In order to extract information on the momentum-space wavefunctions, we can Fourier transform Eqn. (3), but we can also formally invert $\psi(x, t)$ to write

$$
\phi(p, t)=\sum_{n=1}^{\infty} a_{n} \phi_{n}(p) e^{-i E_{n} t / \hbar}
$$

where the momentum-space eigenstates corresponding to the $u_{n}(x)$ are given by

$$
\phi_{n}(p)=\sqrt{\frac{\hbar}{\pi L}}\left(\frac{p_{n}}{p^{2}-p_{n}^{2}}\right)\left\{(-1)^{n} e^{i p L / \hbar}-1\right\}
$$

so that

$$
\langle p\rangle_{t}=\int_{-\infty}^{+\infty} p|\phi(p, t)|^{2} d p
$$

and similarly for other powers of momentum, which will be useful below.

We plot, in Fig. 3, the momentum-space probability densities corresponding to the times in Fig. 2 and note that the wave packet is characterized by a reversal in momentum at $T / 2$ as in Eqn. (12). The initial value of the momentum spread $(\Delta p=10)$ is also recovered at the half-revival, but the uncertainty at many later times is governed not by the intrinsic width of the initial $\phi(p, 0)$ peak, but rather by the 'distance' between the two sharp and well-isolated momentum peaks at approximately $\pm p_{0}$ which gives $\Delta p \approx+p_{0}$ for much of the collapsed phase.

While these examples are somewhat illustrative of the time-dependence of the expectation values and uncertainties in position and momentum variables, we now turn our attention to more detailed examinations of the behavior of $\langle x\rangle_{t}, \Delta x_{t},\langle p\rangle_{t}$, and $\Delta p_{t}$ over three important time scales.

\section{B. Expectation value analysis: short-term, classical behavior}


In many situations, the hierarchy of times given by $\tau<t_{0}<<T$ will hold and we will examine the time-dependence of the wave packet propagation over the first few classical periods. Using our standard parameter set, we calculate the expectation values and spread in position over a time scale equal to the first ten classical periods, $(0,10 \tau)$ and plot the results in Fig. 4. The periodic and almost classical nature of the time-dependence of $\langle x\rangle_{t}$ is clear (compare to Fig. 5 which gives the purely classical result for $x(t), v(t)$ over the same period), but obvious differences are also present. The amplitude of the motion, operationally defined by half the total "left-to-right" distance in $\langle x\rangle_{t}$ (which for a purely classical particle would be constant and equal to L/2) clearly decreases with time in the quantum case and the reason for this is clear from the corresponding plot of the time-dependent spatial uncertainty, $\Delta x_{t}$. The wave packet disperses in time (away from its initial $\Delta x_{0}=0.05$ value, given by the horizontal dashed line) and its width increases, initially at least, in a manner completely consistent with a free particle Gaussian wave packet, as the dotted curve which forms the upper envelope for the solid data is given by Eqn. (18). (In this case, the importance of including the exact quantum time-dependence and not ignoring the dispersion [22] is obvious.) The sharp 'dips' in $\Delta x_{t}$ correspond to 'collisions' of the wave packet with the walls and have been recently studied in the context of a single 'bounce' [23]. As the wave packet gets wider, the value of $\langle x\rangle_{t}$ at the classical collision times decreases (at the right wall) or increases (at the left wall) as the spreading wave packet finds it more difficult to approach the walls. Despite this obvious quantum effect, the short-term time dependence of the system is relatively classical, just as in the Coulomb case.

Turning attention now to the momentum-space behavior, we can make use of Eqn. (23) to evaluate $\langle p\rangle_{t}$ and $\Delta p_{t}$ and we plot these values in Fig. 6 over the first ten classical periods. The correspondence between the sharp classical 'jumps' in the $v(t)$ versus $t$ plot in Fig. 5 and the $t$-dependent quantum expectation value of $p$ in Fig. 6 is clear, but these changes become increasingly 'softer' as the wave packet broadens. The plot of $\Delta p_{t}$ versus $t$ is consistent with expectations from the 'single-bounce' case considered in Ref. [23]. During periods when the packet is not involved in collisions, the momentum spread is initially simply given by the intrinsic width of $\phi(p), \Delta p_{0}$, but increases dramatically during a collision with a wall. At such points, where the momentum distribution is shifting from a single large peak at positive $p=+p_{0}$ to a similar one at $p=-p_{0}$, the spread in momentum values is dominated by the 'distance' between the two peaks. For example, a simple model for the momentum 
distribution at the collision time might be

$$
P_{C L}(p)=\frac{1}{2}\left[\delta\left(p-p_{0}\right)+\delta\left(p+p_{0}\right)\right]
$$

which gives $\langle p\rangle=0$ and $\Delta p=+p_{0}$. This behavior is clearly seen during collisions for times satisfying $t<t_{0}$. The increasing spread in the position-space wave packet (approaching $\Delta p \sim+p_{0}$ ) clearly implies that the momentum distribution comes to include significant components with both $p \approx \pm p_{0}$ for later times as well, giving the same large value of $\Delta p_{t}$ over much of the collapsed phase (as seen earlier in the numerical values in Fig. 3.)

\section{Expectation value analysis: long-term revival behavior}

Turning now to the long-time dependence of the wave packet, we plot, in Fig. $7,\langle x\rangle_{t}$ versus $t$ over one entire revival time $\left(T=800 \tau\right.$.) In the top plot of Fig. 7 , we show $\langle x\rangle_{t}$ for the wave packet at times given by $t=(n+1 / 8) \tau$ and $(n+5 / 8) \tau$ as the solid and dashed curves respectively. (The $t=n \tau$ data yield the horizontal line at $\langle x\rangle_{t}=0.5 L$.) The $(n+1 / 8) \tau$ data (solid curve) corresponds to those times at which the classical particle would be one-half of its way from the center to the right-hand side and classically would always be located at $\langle x\rangle_{t}=0.75 L$, as indicated by the horizontal dashed line: the $(n+5 / 8) \tau$ case would similarly give $\langle x\rangle_{t}=0.25 \mathrm{~L}$ in the classical limit. While the wave packet data are initially consistent with these classical results, they quickly flatten during the collapsed phase so that the expectation value of position at even these times is consistent with $\langle x\rangle=0.5 L$. We note that at $t \approx 800 \tau=T$ the expectation values again match the classical results, which is our first indication of the expected revival or reformation from this expectation value approach.

Similarly, we note that at $t \approx 400 \tau=T / 2$, we find that the expectation values for position have reversed themselves, consistent with Eqn. (8) for the half-revival. In the lower half of Fig. 7, we show results for $\langle x\rangle_{t}$ for all times in the range $t=(116 \tau, 164 \tau)$ to illustrate the fine structure apparent in the time-dependence and how the wave packet oscillates, with very small excursions, around $\langle x\rangle_{t}=0.5 \mathrm{~L}$ during most of the collapsed phase.

In order to further probe the manner in which the probability distribution 'flattens' during the collapse and reforms during a revival, we plot in Fig. 8 the uncertainty $\Delta x_{t}$ versus time over the same time periods as in Fig. 7: we show values for times given by $t=n \tau$ only. To compare the results to a putative flat probability distribution, we note that this case would 
be given by

$$
P_{\text {flat }}(x)=\frac{1}{L}
$$

and would yield expectation values

$$
\langle x\rangle_{\text {flat }}=\frac{L}{2} \quad\left\langle x^{2}\right\rangle_{\text {flat }}=\frac{L^{2}}{3} \quad \Delta x_{\text {flat }}=\frac{L}{\sqrt{12}}=0.288 L
$$

We note that $\Delta x_{t}$ grows (consistent with Eqn. (18)), but rapidly flattens out during much of the collapsed phase at a value which is indeed consistent with $\Delta x_{\text {flat }}=0.288 \mathrm{~L}$ over much of the entire revival period. (At the bottom of Fig 8, we show the behavior of $\Delta x_{t}$ for all times in the interval $(116 \tau, 164 \tau)$ indicating the oscillations around the expected 'flat' result.)

The revivals at $T=800 \tau$ and $T / 2=400 \tau$ are also apparent here as the uncertainty returns to its initial value, indicating a reformation of the initial wave packet, perhaps at a different location. We also note, however, the additional feature of quasi-revivals at $t=200 \tau$ and $600 \tau(T / 4,3 T / 4)$ where the wave packet returns to its initial width. To illustrate the nature of the wave packet at these times, we have already shown in Fig. 2 the form of the packet near $t \approx 200 \tau$. The wave packet at $t=200 \tau$ does not precisely return to its initial state (the real part of $\psi(x, T / 4)$ is larger than $\psi(x, 0)$, while the imaginary part of $\psi(x, T / 4)$ vanishes), but does so in a way which reproduces $\Delta x_{t=T / 4}=\Delta x_{0}$. We can see that this is a very special case, as the $t=\tau / 8$ wave packet is obviously split into two pieces at $t=\tau / 8+T / 4$ as it is 'half way' to being reformed at the half-revival time. We can also note that at $t=100 \tau, 300 \tau, 500 \tau$, and $700 \tau$ that there are 'anti-revivals' in that the wave packets are spread somewhat more than during the rest of 'flat phase' of the collapsed phase. The position-space wavefunction at $t=100 \tau$ was also shown in Fig. 2 to illustrate this 'anti-correlation'.

We can also visualize the long-time behavior of the momentum-space variables by plotting $\langle p\rangle_{t}$ and $\Delta p_{t}$ versus $t$ over one revival time in Fig. 9 (again, for values of $t=n \tau$.) We note that the momentum spread quickly saturates at the 'flat' value of $\Delta p=+p_{0}$, except when it returns for revivals at $t=T / 2$ and $T$. The expectation value of momentum reverses sign at $t=T / 2$, as expected from Eqn. (12), while returning to its original value only at the standard revival when $t=T$.

While we have explicitly considered only the quasi-Gaussian wavepacket with specific numerical values here, many of the same effects are seen quite generally. Changes in most of the parameters simply change the appropriate time scales involved. Wave packets with 
different initial positions, $x_{0} \neq L / 2$, do not exhibit the special quasi-revivals at $t=T / 4,3 T / 4$ because they lack the required additional symmetry. Different functional forms for the expansion coefficients (the discrete versions of Lorentzian momentum weightings or initially flat spatial wave packets, for example) all exhibit similar behavior to that discussed here.

\section{Expectation value analysis: medium-term, collapse behavior}

One aspect of the behavior of wave packets in the infinite well which has not been examined in much detail as has the pattern of revivals (fractional or otherwise) is the decay phase, the time over which the spreading of the wave packet leads to an increasing lack of coherence (as measured by $C(t)$ ) and because of which the position-space probability density approaches the 'flat' distribution observed by Segre and Sullivan [10]. One measure of a decay time scale associated with this spreading is given by the scaling behavior of $|C(t)|$ at integral values of the classical period, namely $t=n \tau$. For the oscillator, for example, we have $|C(t=n \tau)|=1$ since the wave packet motion reforms exactly with the classical oscillation frequency. For the infinite well, we find (after a large number of numerical trials) that the autocorrelation function for such integral times is initially given by

$$
|C(t=n \tau)| \sim e^{-\left(n \tau / T_{C}\right)^{2}} \quad \text { for } \quad n \tau \lesssim T_{C}
$$

where the collapse time, $T_{C}$, is given by

$$
T_{C}=\frac{T}{2 \pi \Delta n^{2}}=4 t_{0}=4\left(\frac{2 m\left(\Delta x_{0}\right)^{2}}{\hbar}\right)
$$

and where $\Delta n$ is the dispersion in the $n$-distribution for the Gaussian wave packet. This result may not be unexpected since the decrease in coherence measured by $C(t)$ is directly tied to the spreading of the wavepacket and $t_{0}$ is therefore the natural time scale which arises in an autocorrelation analysis of this type.

Examination of Fig. 8, however, suggests that there is a second scale involved in the decay process, namely that associated with the time it takes for the wave packet to approach the spatially flat probability distribution. In order to better visualize the approach to the 'flat' distribution, we plot $\Delta x_{t}$ versus $t$ in the range $(0, T / 8=100 \tau)$ for three different values of $\Delta x_{0}$ in Fig. 10. We note that in each case the uncertainties initially follow the free-particle Gaussian result in Eqn. (18), but then level off to the same 'flat' value of $\Delta x_{\text {flat }}=L / \sqrt{12}$. 
We can estimate the time it takes to reach the constant value during the collapse phase by equating

$$
\frac{L}{\sqrt{12}}=\Delta x_{\text {flat }}=\Delta x_{t}=\Delta x_{0} \sqrt{1+\left(\frac{t}{t_{0}}\right)^{2}}
$$

which gives

$$
t_{\text {flat }} \approx t_{0}\left(\frac{L}{\sqrt{12} \Delta x_{0}}\right)=\frac{1}{\sqrt{6 \pi}}\left(T t_{0}\right)^{1 / 2}=\frac{8}{\sqrt{12}}\left(\frac{m L \Delta x_{0}}{\hbar}\right)
$$

which defines still another time scale, typically an intermediate scale between $t_{0}$ and $T$. Numerical studies, such as in Fig. 10, indicate that the position-space uncertainty saturates at its 'flat' value at a time given roughly by $2 t_{\text {flat }}$. The three cases shown in Fig. 10 then illustrate that this flattening time does indeed scale with $\Delta x_{0}$, in contrast to the coherence decay time scale $T_{C}$ which is proportional to $\Delta x_{0}^{2}$. We can see the same flattening time scale (and its proportionality to $\Delta x_{0}$ ) by plotting the medium-scale time dependence of $\langle x\rangle_{t},\langle p\rangle_{t}$,

and $\Delta p_{t}$ in Fig. 11, all of which approach the appropriate 'flat' value with a time scale of roughly $2 t_{\text {flat }}$. It is not immediately apparent how information on this important time scale is readily obtainable from an autocorrelation analysis (for example, as shown in Fig. 1), providing more evidence for the usefulness of this more intuitive approach.

\section{E. Conclusions}

We have studied the time-dependence of wave packets in the infinite well, their collapse and revival structure, and the time scales appropriate for each phase by examination of the expectation values and uncertainties in the familiar variables of one-dimensional quantum mechanics, position and momentum. The visualizations provided here complement more formal derivations of revival structures studied using autocorrelation function analyses. In the case of the approach to the collapsed phase of the wave packet approaching a 'flattened' state, this type of expectation value analysis very naturally yields information on the scaling properties of the natural time scale for the collapse, in contrast to an autocorrelation function approach where such information is not so immediately and intuitively obvious.

\section{Acknowledgments}


This work was supported in part by the National Science Foundation under Grant DUE9950702. We are grateful for helpful comments made by R. Diehl, M. Doncheski, and J. Yeazell as well as for the very useful advice of a referee. 


\section{Appendix}

Motivated by the simple revival structure observed for the harmonic oscillator and infinite well potentials in Ref. [11], we present here a brief analysis of the revival time for the general, one-dimensional power-law potential

$$
V_{(k)}(x) \equiv V_{0}\left|\frac{x}{a}\right|^{k}
$$

which interpolates between these two special cases for $k=2$ (oscillator) and $k=\infty$ (infinite square well). While there is really only one independent dimensionful parameter in this potential, namely $V_{0} / a^{k} \equiv \alpha$, we write it in this form to emphasize the $k \rightarrow \infty$ limit where we obtain an infinite well (of width $2 a$, compared to our earlier analysis.) A simple variation is the 'half' power-law potential given by

$$
\tilde{V}_{(k)}(x)= \begin{cases}V_{(k)}(x) & \text { for } x>0 \\ 0 & \text { for } x<0\end{cases}
$$

which is then appropriate for the analysis of the 'quantum bouncing ball' discussed recently in Ref. [19] when $k=1$ and $V_{0} / a \equiv m g$.

In order to make use of an autocorrelation analysis as in Ref. 11], we require the quantized energy eigenvalues, $E_{n}^{(k)}$. Since we are dealing with situations in which $n>>1$, it will be a good approximation to utilize the WKB energy quantization condition to evaluate the $E_{n}^{(k)}$. The standard version (which assumes wave-function matching at linear walls via Airy functions) is given by

$$
\int_{-x_{0}}^{+x_{0}} \sqrt{2 m\left[E_{n}^{(k)}-V_{(k)}(x)\right]} d x=\left(n+\frac{1}{2}\right) \hbar \pi
$$

with $n=0,1,2, \ldots$ and where

$$
\pm x_{0}= \pm\left(\frac{E_{n}}{V_{0}}\right)^{1 / k} a
$$

are the classical turning points. This expression is known to be exact for the harmonic oscillator. When the boundary conditions at a given turning point are imposed by infinite walls where the wavefunction must actually vanish, the appropriate matching coefficients are $C_{L}, C_{R}=1 / 2$ instead of the $C_{L}, C_{R}=1 / 4$ for linear walls [24], so that for the infinite square well the right hand side is replaced by $(n+1 / 2) \rightarrow(n+1)$ which also gives the exact 
answer for this case. (The combination of the two to describe the 'half oscillator' where $V(x)=m \omega^{2} x^{2} / 2$ for $x>0$ and an infinite wall at the origin is then also given exactly with these two cases.) Using the standard WKB form for all $k<\infty$, we find the energy quantization condition [18]

$$
E_{n}^{(k)}=\left[(n+1 / 2) \frac{\hbar \pi}{2 a \sqrt{2 m}} V_{0}^{1 / k} \frac{\Gamma(1 / k+3 / 2)}{\Gamma(1 / k+1) \Gamma(3 / 2)}\right]^{2 k /(k+2)}
$$

(where $(n+1 / 2) \rightarrow(n+1)$ for $k \rightarrow \infty$.) This reproduces the exact oscillator and infinite well examples in the $k=2$ and $k \rightarrow \infty$ limits. The classical periods for a given value of $k$ and $n$ will then be given by

$$
\tau_{n}^{(k)}=\frac{2 \pi \hbar}{E_{n}^{(k)}}(n+1 / 2)\left(\frac{2+k}{2 k}\right) \equiv \tau(k, n)
$$

which reduces to the familiar oscillator and infinite well results. Using the result of Ref. [11] for the revival time, namely

$$
T=\frac{4 \pi \hbar}{d^{2} E_{n} / d n^{2}}=\frac{4 \pi \hbar}{E_{n}^{\prime \prime}}
$$

we find that the revival times are given by the simple expression

$$
T_{\text {rev }}=T(k, n)=\left|\frac{k+2}{k-2}\right| 2 n \tau(k, n)
$$

This can be confirmed by explicit evaluation of $|C(t)|,|\bar{C}(t)|$ over one revival time for any value of $k>0$ where one finds plots which are very similar to Fig. 1, except that the revivals are no longer exact as they are in the case of the infinite well. This form explicitly exhibits the divergence we expect in the oscillator case (when $k \rightarrow 2$ ) where the wave packets are exactly periodic [25], [26].

We have also performed a more numerical analysis using the quasi-Gaussian wave packet to examine the short-time decay in correlations as measured by the decrease in the magnitude of $|C(t)|$. When we evaluate the autocorrelation function at integral values of the classical period, we again find that

$$
|C(t=n \tau)| \sim e^{-\left(n \tau / T_{C}\right)^{2}}
$$

where

$$
T_{C} \equiv\left[\left|\frac{k+2}{k-2}\right| 2 n \tau(k, n)\right] \frac{1}{2 \pi \Delta n^{2}}=\frac{T(k, n)}{2 \pi \Delta n^{2}}
$$

which reproduces the result for the infinite well we have considered more explicitly, as well as exhibiting the appropriate divergence as $k \rightarrow 2$ as we approach the exactly periodic 
oscillator case. This result also holds for other, non-Gaussian wave packet expansions, with the numerical factor $2 \pi$ replaced by different numerical coefficients of the same order. Two open questions which would be interesting to explore further in this context of the general well are whether during the collapsed phase the position-space probability naturally approaches a semi-classical probability density (as in Ref. 27]) over much of the collapsed phase and, if so, how the 'flattening' time to approach this semi-classical distribution scales with $k$ and other parameters, i.e., what is the generalization of Eqn. (32).

The case of the 'half' general-power law potential given by Eqn. (34) is easily analyzed in the same manner. The WKB energy quantization condition is applied at the classical turning points $x=+x_{0}$ and $x=0$ with the result

$$
\tilde{E}_{n}^{(k)}=\left[(n+3 / 4) \frac{\hbar \pi}{a \sqrt{2 m}} V_{0}^{1 / k} \frac{\Gamma(1 / k+3 / 2)}{\Gamma(1 / k+1) \Gamma(3 / 2)}\right]^{2 k /(k+2)}
$$

with the factor $(n+3 / 4) \rightarrow(n+1)$ for $k \rightarrow \infty$. (Recall the discussion after Eqn. (36) about the appropriate matching coefficients for infinite wall boundaries.) The classical period is then given by Eqn. (38) with $E_{n}^{(k)} \rightarrow \tilde{E}_{n}^{(k)}$ and $(n+1 / 2) \rightarrow(n+3 / 4)$. We find that the same relationship between revival time and classical period as in Eqn. (40) holds and we note that our general result then reproduces the revival time found in the special case of the 'quantum bouncer' considered in Ref. [19] when $k=1$ and $V_{0} / a=m g$.

Gea-Banacloche [19] has also discussed a collapse time for the case of the 'quantum bouncing ball' (the $k=1$ case of the 'half-well') and we note in passing that his expression is close in spirit to the 'flattening' time discussed for the infinite well as his expression for the collapse time scales as $1 / \Delta n$ as does $t_{\text {flat }}$, and not, for example, like $T_{C} \propto 1 / \Delta n^{2}$ for the decay time obtained by the scaling properties of the autocorrelation function. 
[1] A. Goldberg, H. M. Schey, and J. L. Schwartz, "Computer-generated motion pictures of onedimensional quantum-mechanical transmission and reflection phenomena", Am. J. Phys. 35, 177-186 (1967).

[2] M. H. Bramhall and B. M. Casper, "Reflections on a wave packet approach to quantum mechanical barrier penetration", Am. J. Phys. 38, 1136-1145 (1970); J. R. Merrill, "The propagation of quantum mechanical wave packets", Am. J. Phys. 41, 1101-1103 (1973); J. S. Boleman and S. B. Haley, "More time-dependent calculations for the Schrödinger equation", Am. J. Phys. 43, 270-271 (1975); C. L. Hammer, T. A. Weber, and V. S. Zidell, "Time-dependent scattering of wave packets in one dimension", Am. J. Phys. 45, 933-941 (1977); B. Diu, ”Plane waves and wave packets in elementary quantum mechanics problems", Eur. J. Phys. 1, 231240 (1980); A. Edgar, "Reflection of wave packets from a quantum well with a tunneling transmission resonance", Am. J. Phys. 63, 136-141 (1995).

[3] P. A. Deutchman, "Tunneling between two square wells - Computer movie", Am. J. Phys. 39, 952-954 (1971). E. A. Johnson and H. Thomas Williams, "Quantum solutions for a symmetric double square well", Am. J. Phys. 50, 239-243 (1981).

[4] I. Galbraith, Y. S. Ching, and E. Abraham, "Two-dimensional time-dependent quantummechanical scattering event", Am. J. Phys. 52, 60-68 (1984).

[5] J. C. Hamilton, J. L. Schwartz, and W. A. Bowers, "Computer generated films for solid state physics", Am. J. Phys. 40, 1657-1661 (1972); G. Friedmann and W. A. Little, "A study of the wave function of a particle striking a crystal interface", Am. J. Phys. 61, 835-843 (1993).

[6] See, for example, J. R. Hiller, I. D. Johnston and D. F. Styer, Quantum Mechanics Simulations: The Consortium for Upper-Level Physics Software (New York, Wiley, 1995).

[7] L. S. Brown, "Classical limit of the hydrogen atom", Am. J. Phys. 41 525-530 (1972).

[8] M. Nauenberg, "Quantum wave packets on Kepler elliptic orbits", Phys. Rev. A40, 1133-1136 (1989); I. M. Suarez Barnes, M. Nauenberg, M. Nockleby, and S. Tomsovic, "Semiclassical theory of quantum propagation: the Coulomb potential", Phys. Rev. Lett. 71, 1961-1964 (1993).

[9] M. Nauenberg, C. Stroud, and J. Yeazell, "The classical limit of an atom", Sci. Am. 270, 4449 (1994); J. Parker and C. R. Stroud, Jr., "Coherence and decay of Rydberg wave packets", 
Phys. Rev. Lett. 56, 716-719 (1986); J. A. Yeazell, M. Mallalieu, and C. R. Stroud, Jr. "Observation of the collapse and revival of a Rydberg electronic wave packet", Phys. Rev. Lett. 64, 2007-2010 (1990); For a review, see G. Alber and P. Zoller, "Laser excitation of electronic wave packets in Rydberg atoms", Phys. Rep 199, 231-280 (1991).

[10] C. U. Segre and J. D. Sullivan, "Bound-state wave packets", Am. J. Phys. 44, 729-732 (1976).

[11] R. Bluhm, V. Alan Kostelecký, and James A. Porter, "The evolution and revival structure of localized wave packets", Am. J. Phys. 64, 944-953 (1996).

[12] D. L. Aronstein and C. R. Stroud Jr, "Fractional wave-function revivals in the infinite square well", Phys. Rev. A55, 4526-4537 (1997).

[13] I. Sh. Averbukh and N. F. Perelman, "Fractional revivals: universality in the long-term evolution of quantum wave packets beyond the correspondence principle dynamics", Phys. Lett. A139, 449-454 (1989);

[14] M. Nauenberg, "Autocorrelation function and quantum recurrence of wavepackets", J. Phys. B. At. Mol. Opt. Phys. 23, L385-390 (1990).

[15] The revival structure in the two-dimensional infinite well, with non-commensurate sides, leading to a non-trivial energy eigenvalue structure has also been analyzed using these techniques. See R. Bluhm, V. Alan Kostelecký, and B. Tudose, "Wave-packet revivals for quantum systems with nondegenerate energies", Phys. Lett. A222, 220-226 (1996); G. S. Agarwal and J. Banerji, "Fractional revivals in systems with two times scales", Phys. Rev. A57 3880-3884 (1998).

[16] P. Stifter, W. E. Lamb Jr, and W. P. Schleich, "The particle in a box revisited", in Frontiers of Quantum Optics and Laser Physics, Proceedings of the International Conference on Quantum Optics and Laser Physics (Springer, Singapore, 1997) edited by S. Y. Zhu, M. S. Zubairy, and M. O. Scully, pp. 236-246.

[17] D. F. Styer, "The motion of wave packets through their expectation values and uncertainties", Am. J. Phys. 58, 742-744 (1989).

[18] P. Sukhatme, "WKB energy levels for a class of one-dimensional potentials", Am. J. Phys. 41, 1015-1016 (1973).

[19] J. Gea-Banacloche, "A quantum bouncing ball", Am. J. Phys. 67 776-782 (1999).

[20] R. L. Liboff, Introductory Quantum Mechanics (Reading, Addison-Wesley, 1991) 2nd edition, pp. $555-556$. 
[21] R. W. Robinett, Quantum Mechanics: Classical Results, Modern Systems, and Visualized Examples (New York, Oxford University Press, 1997), pp. 60-61.

[22] J. V. Greenman, "Non-dispersive mirror wave packets", Am. J. Phys. 40, 1193-1201 (1972).

[23] M. A. Doncheski and R. W. Robinett, "Anatomy of a quantum 'bounce"', Eur. J. Phys. 20, 29-37 (1999).

[24] A. Migdal and V. Krainov, Approximation Methods in Quantum Mechanics (W. A. Benjamin, New York, 1969), pp. 111-123.

[25] D. S. Saxon, Elementary Quantum Mechanics (New York, McGraw-Hill, 1968) pp. 144-147.

[26] For a large number of different ways of deriving the harmonic oscillator propagator, see B. Holstein, Am. J. Phys. 66, 583 (1998).

[27] R. W. Robinett, "Quantum and classical probability densities for position and momentum", Am. J. Phys. 63, 823-832 (1995). 


\section{Figure Captions}

Fig. 1. Plot of the autocorrelation function, $|C(t)|$, (in Eqn. (11) ) for the infinite square well. The modulus of $C(t)$ is evaluated at integral values of $t / \tau$ over the first half revival time; the curves are symmetric about $t=T / 2=400 \tau$. The vertical dashed lines indicate values of some of the possible fractional revivals, namely $(p / q) T$ for integral values of $p<q$. The standard wave packet parameters in Eqns. (14), (20), and (21) are used. The related correlation function, $\bar{C}(t)$, defined in Eqn. (22), is also plotted and shows much of the same structure, including the 'anti-revival' at $t=T / 2=400 \tau$ where the wave packet reforms, but with opposite momentum values.

Fig. 2. Position-space probability distributions, $|\psi(x, t)|^{2}$ versus $x$, for the quasi-Gaussian wave packet at various times. The top panel shows the wave packet at and just after the initial time. The $t=100 \tau$ plot is typical of a fractional revival where the wave packet reforms into a small number of recognizable features. The $t=124 \tau$ is typical of a more random later time when the wavefunction approaches the 'flat' probability distribution, $P_{\text {flat }}(x)=1 / L$, indicated by the horizontal arrow. The $t \approx 200 \tau$ cases show a special fractional revival where an initially central $\left(x_{0}=0\right)$ wave packet reforms with the initial value of $\Delta x_{0}$. Finally, the reformation of the $t=0$ wave packet at the half-revival time, $t=T / 2=400 \tau$ is indicated, as is the reformation of the $t=\tau / 8$ packet, but flipped about the center of the well as in Eqn. (91).

Fig. 3. Momentum-space probability distributions, $|\phi(p, t)|^{2}$ versus $p$, corresponding to the sample times shown in Fig. 2; the vertical dashed lines indicate the values $p= \pm p_{0}=$ $\pm n_{0} \pi \hbar / L= \pm 400 \pi$. Note the reversal in momentum values at the half-revival time, $t=T / 2=400 \tau$.

Fig. 4. Average value for position, $\langle x\rangle_{t}$ (top), and spread in position, $\Delta x_{t}$ (bottom), for the quasi-Gaussian wavepacket defined by the parameters in Eqns. (14), (20), and (21) over a time interval corresponding to the first ten classical periods. In the bottom figure, the first three multiples of the spreading time, $t_{0}$, are shown (vertical dashed lines). The horizontal dashed line corresponds to the initial spread, $\Delta x_{0}=0.05$. The dotted curve is given by the expression for the time-dependent $\Delta x_{t}$ for free-particle Gaussian wavepackets given by Eqn. (18). 
Fig. 5. Classical motion $(x(t)$ and $v(t)$ versus $t)$ of a point particle in an infinite square well potential, starting at the center of the well with constant speed $v_{0}$, moving in the positive- $x$ direction.

Fig. 6. Same as Fig. 4, but for the average value of momentum, $\langle p\rangle_{t}$ (top), and spread in momentum, $\Delta p_{t}$ (bottom). In the bottom plot, the initial momentum spread, $\Delta p_{0}$, is shown as a horizontal dashed line, as is the maximum value, $\Delta p=+p_{0}$, corresponding roughly to two equal peaks in $|\phi(p, t)|^{2}$ at $p= \pm p_{0}$ as in Eqn. (26).

Fig. 7. Average value of position, $\langle x\rangle_{t}$ versus $t$, over one revival time $(T=800 \tau)$. The average value of position for $t=(n+1 / 8) \tau$ (solid) and $(n+5 / 8) \tau$ (dashed) are plotted in the top figure. (For values given by $t=n \tau$, one obtains the horizontal solid line corresponding to $\langle x\rangle_{t}=0.5 L$.) The revival at $t=T=800 \tau$ where expectation values return to their $t \approx 0$ values is evident, as is the half-revival at $t=T / 2=400 \tau$ where the expectation values are mirrored about the center of the well as predicted by Eqn. (8). The value of $\langle x\rangle_{t}$ for all times during the interval $(116 \tau, 164 \tau)$ is shown on the bottom plot, indicating the small oscillations about the 'flat' value of $\langle x\rangle=0.5 L$ during most of the collapsed phase.

Fig. 8. Uncertainty or spread in position, $\Delta x_{t}$ versus $t$ (evaluated at times given by $t=n \tau$ ) over one revival time $(T=800 \tau)$. The 'flat' value of $\Delta x_{\text {flat }}=L / \sqrt{12} \approx 0.288 L$ is shown as the dashed horizontal line. The spread in position initially follows the dashed curve (indicated by $\Delta x_{\text {free }}$ ) given by the free-particle expression in Eqn. (18), but then 'turns over' and approaches the 'flat' value. The time interval $(116 \tau, 164 \tau)$ is also shown to indicate the oscillations around the 'flat' value during the collapsed phase. The full revival at $t=T=800 \tau$ as well as the half-revival at $t=T / 2=400 \tau$ are evident, as are the special quasi-revivals at $T / 4=200 \tau$ and $3 T / 4=600 \tau$ which appear in the $x_{0}=L / 2$ case only.

Fig. 9. Average value $\left(\langle p\rangle_{t}\right.$ versus $\left.t\right)$ and uncertainty $\left(\Delta p_{t}\right.$ versus $\left.t\right)$ in momentum for the quasi-Gaussian wave packet over one revival time. The reversal in momentum seen in $\langle p\rangle_{t}$ and the reformation of the wave packet to its initial width are obvious at the half-revival time, $t=T / 2=400 \tau$. The spread in momentum corresponding to the 'flat' distribution of probability in position-space $\left(\Delta p=+p_{0}\right)$ is also evident over a 
large fraction of the collapsed phase.

Fig. 10. Plot of $\Delta x_{t}$ versus $t$ showing the approach to the 'flat' distribution for various values of the initial spread, $\Delta x_{0}$. In each case, the curves initially follow the free-particle Gaussian expression in Eqn. (18), but then 'turn over' and saturate at $\Delta x=L / \sqrt{12}$.

Fig. 11. Plots of $\langle x\rangle_{t}$ (top), $\langle p\rangle_{t}$ (middle), and $\Delta p_{t}$ (bottom) showing the approach to the values of these quantities corresponding to the 'flat' distribution. Note that the characteristic time for flattening is the same in all three cases and that it scales as indicated in Eqn. (32), namely $t_{\text {flat }} \propto \Delta x_{0}$. 
:I DIH

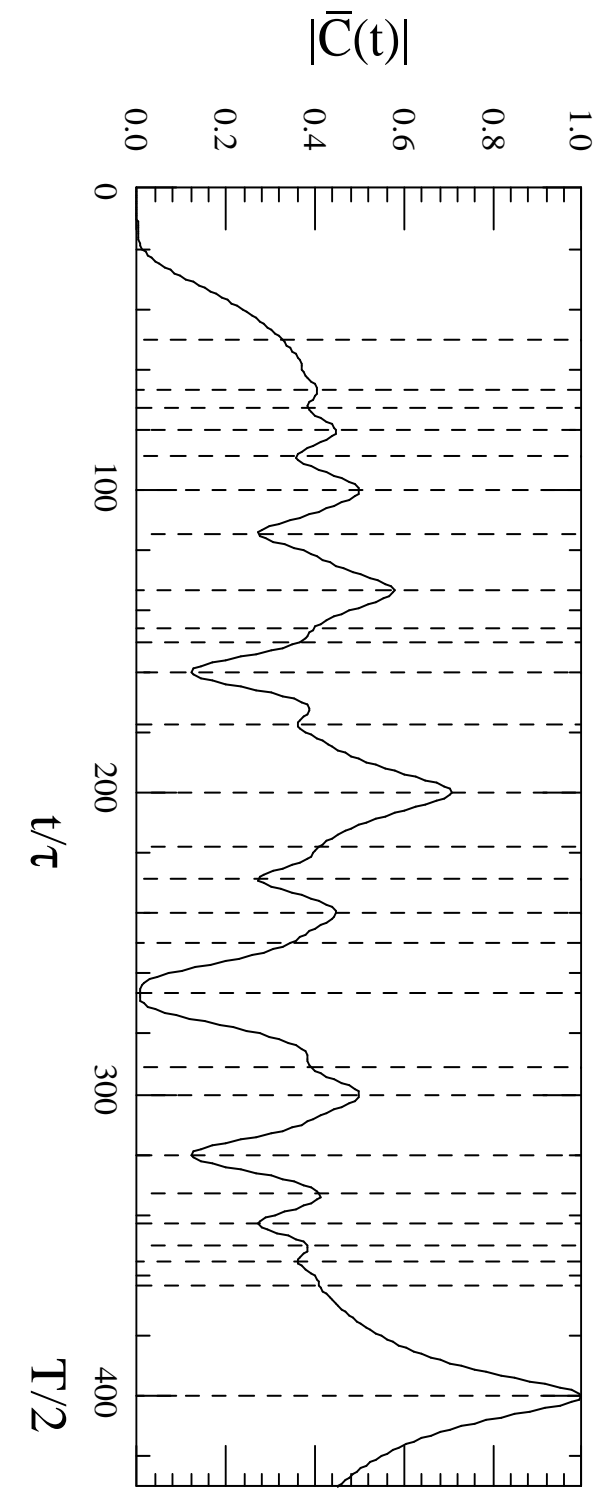

$|\mathrm{C}(\mathrm{t})|$
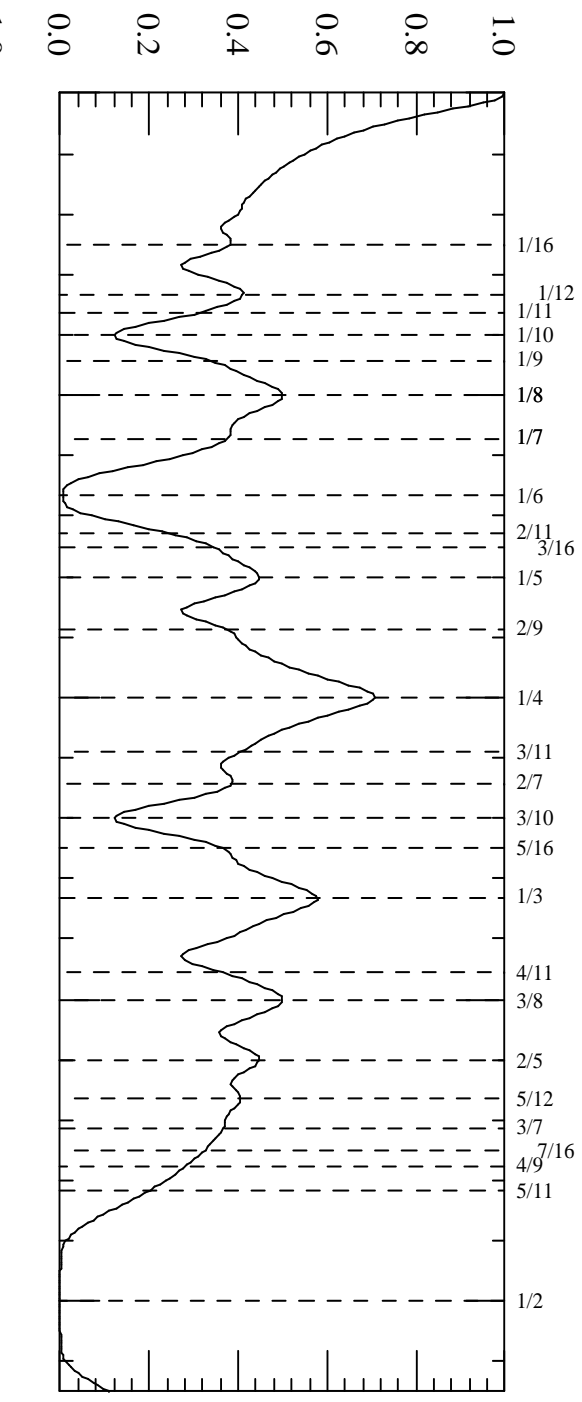


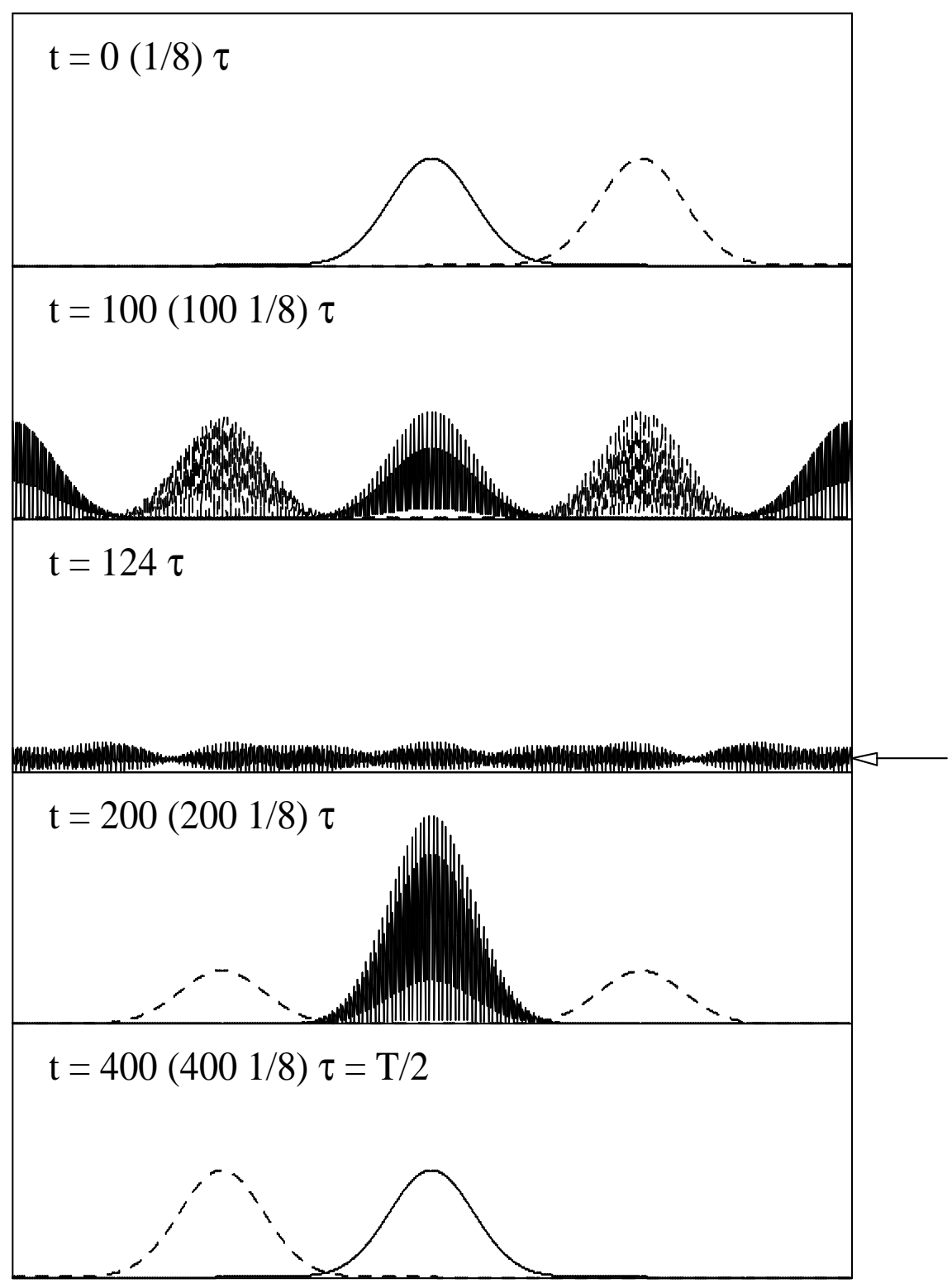

FIG. 2: 


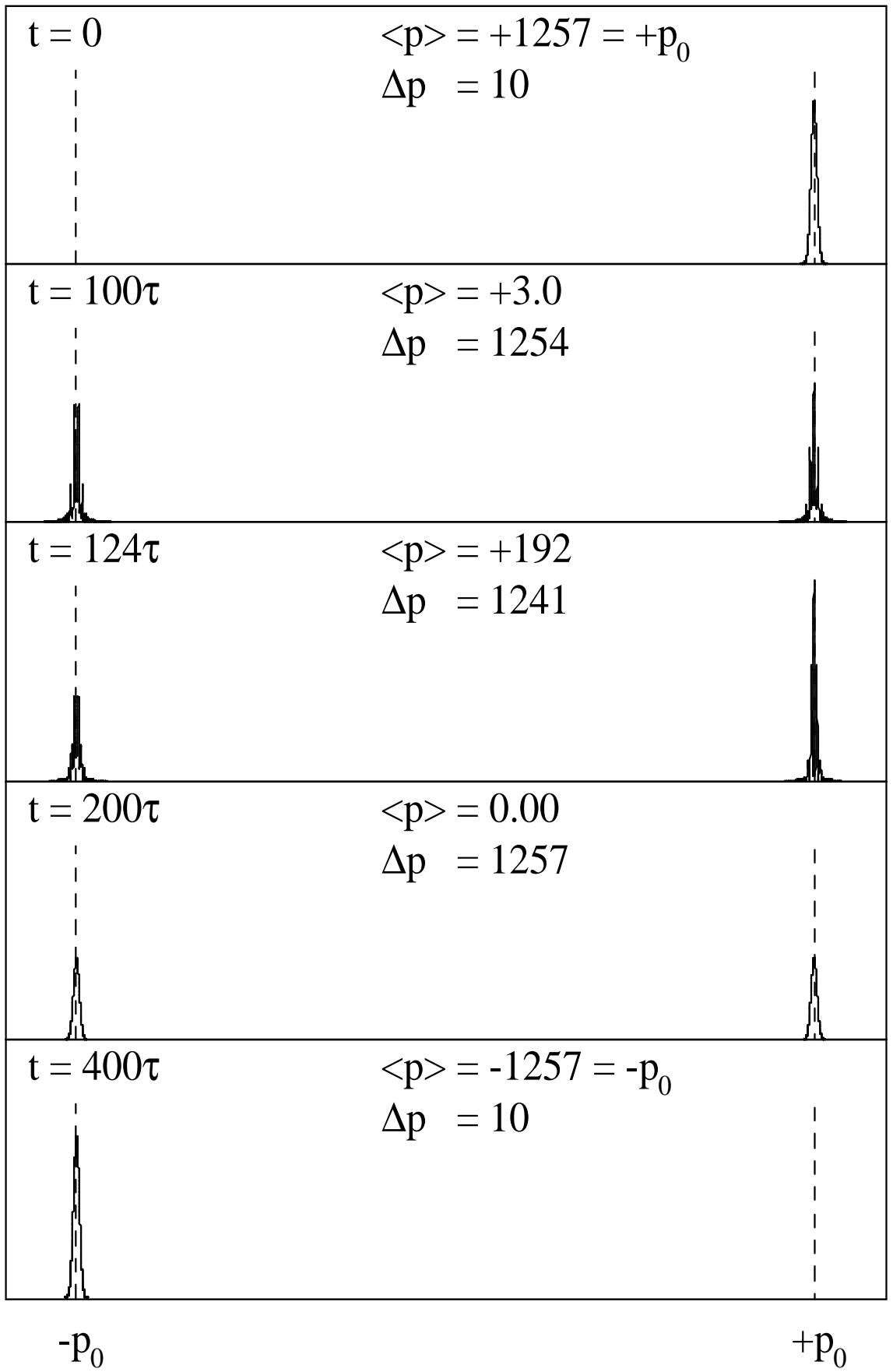

FIG. 3: 


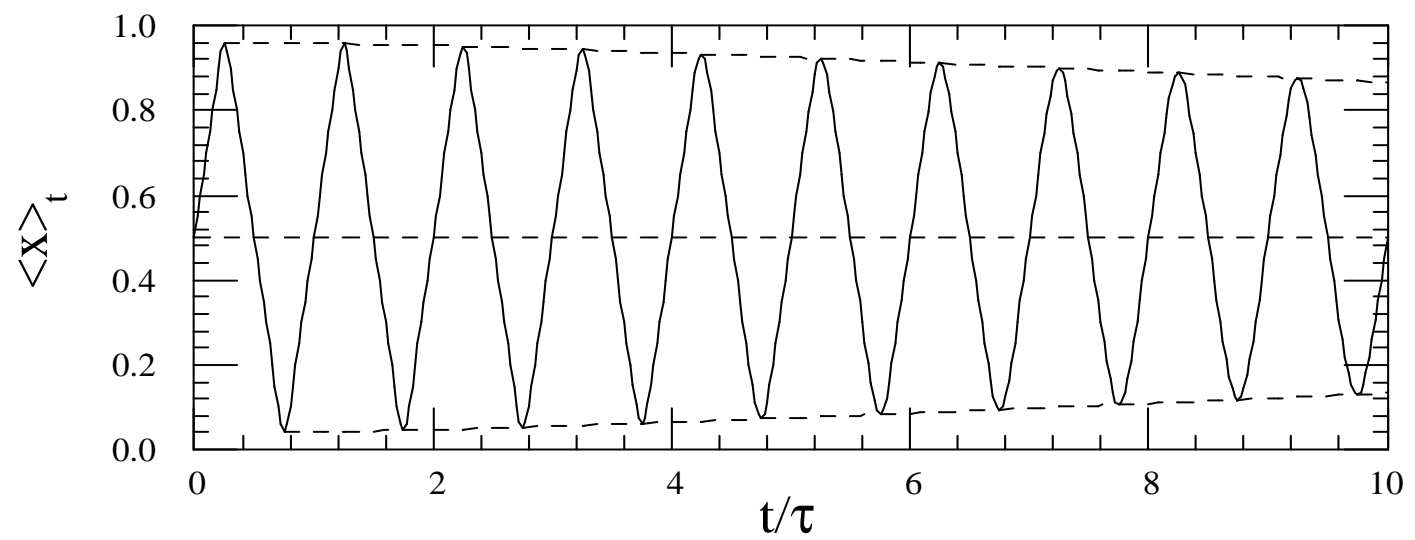

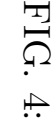

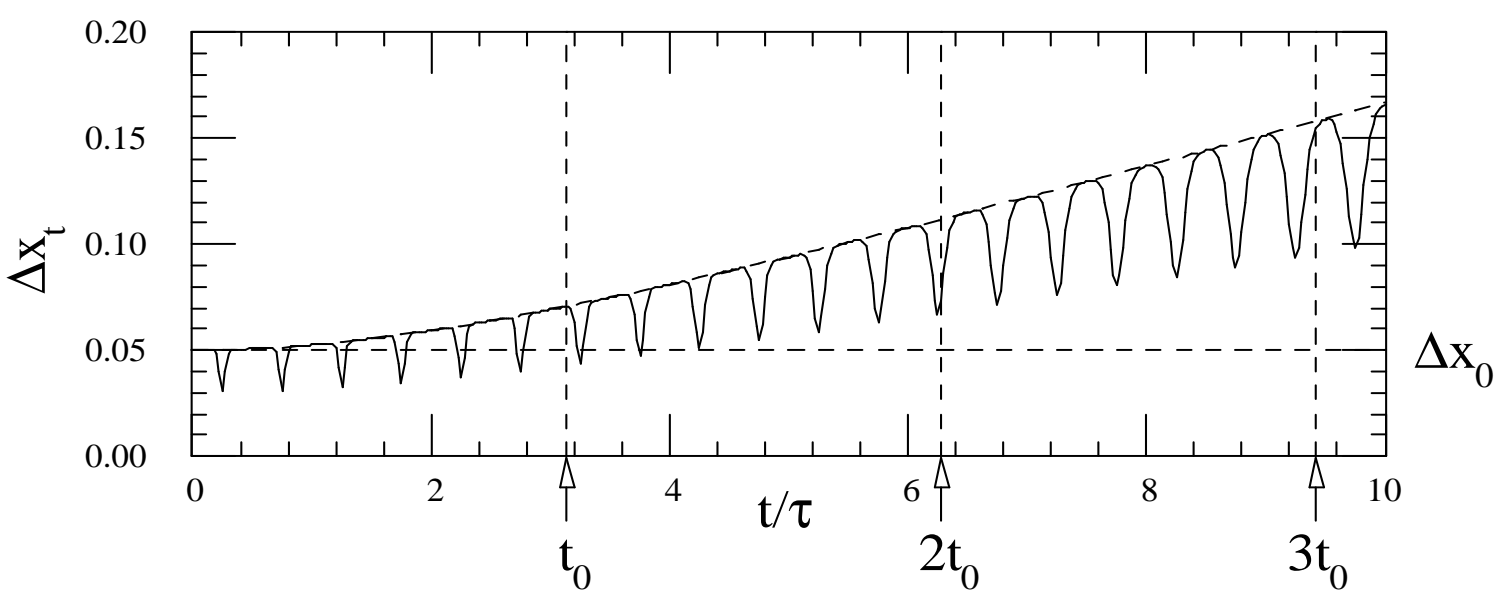




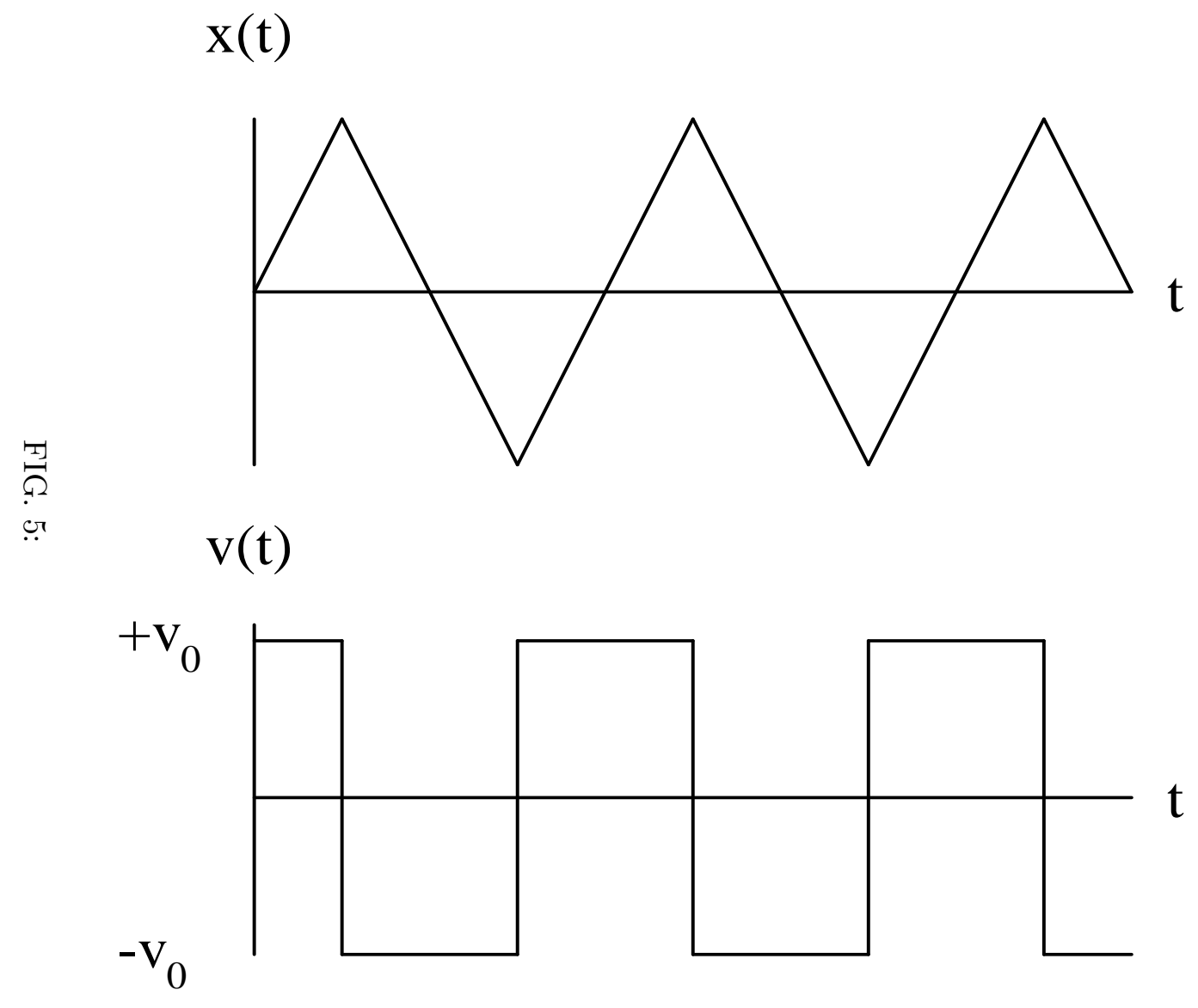




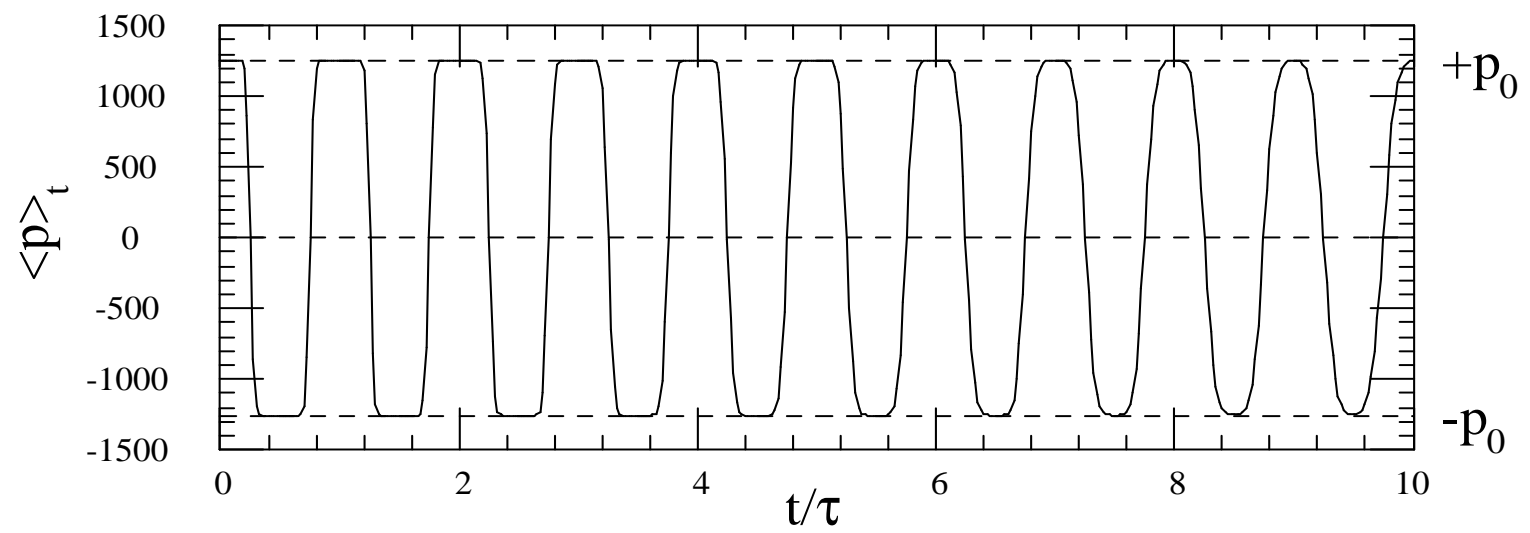

T:
0
0

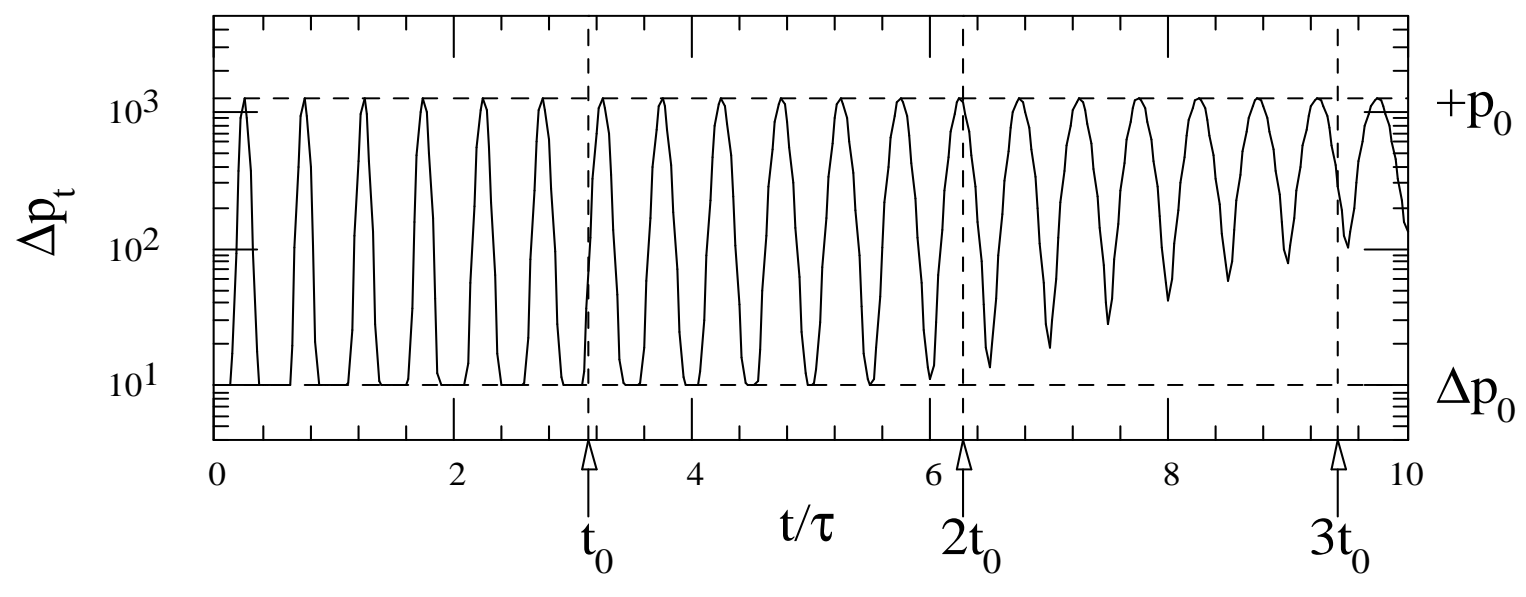




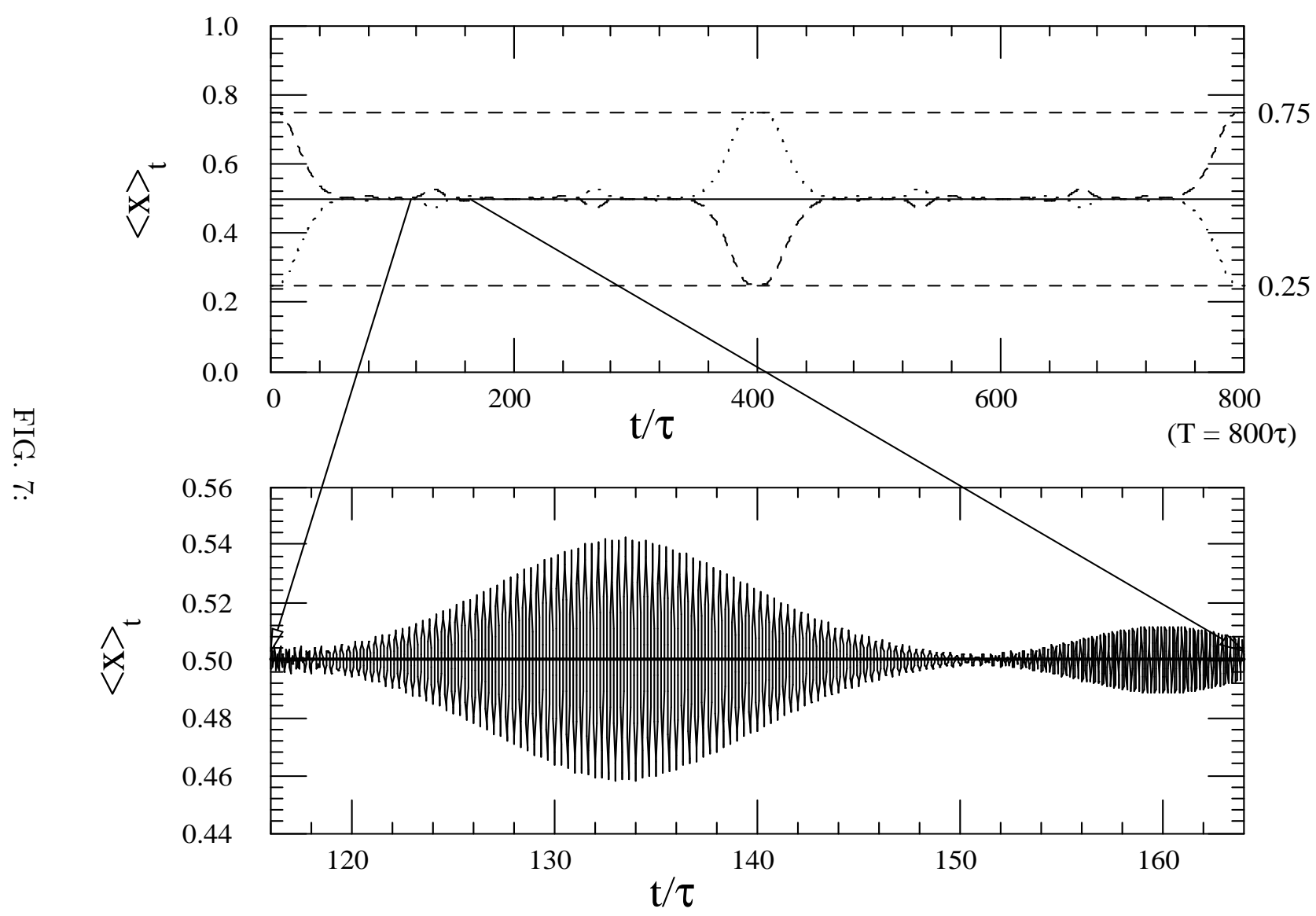




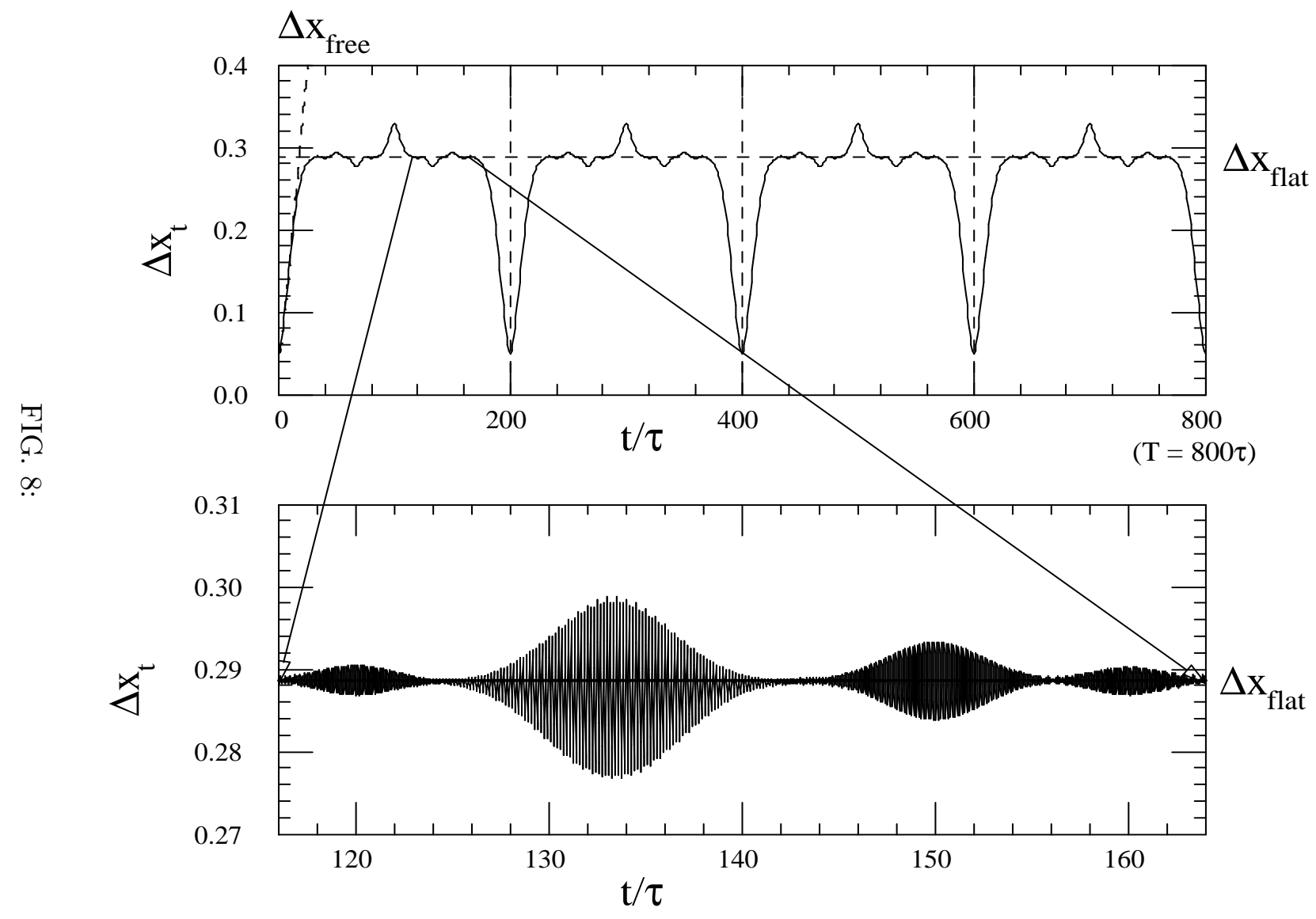




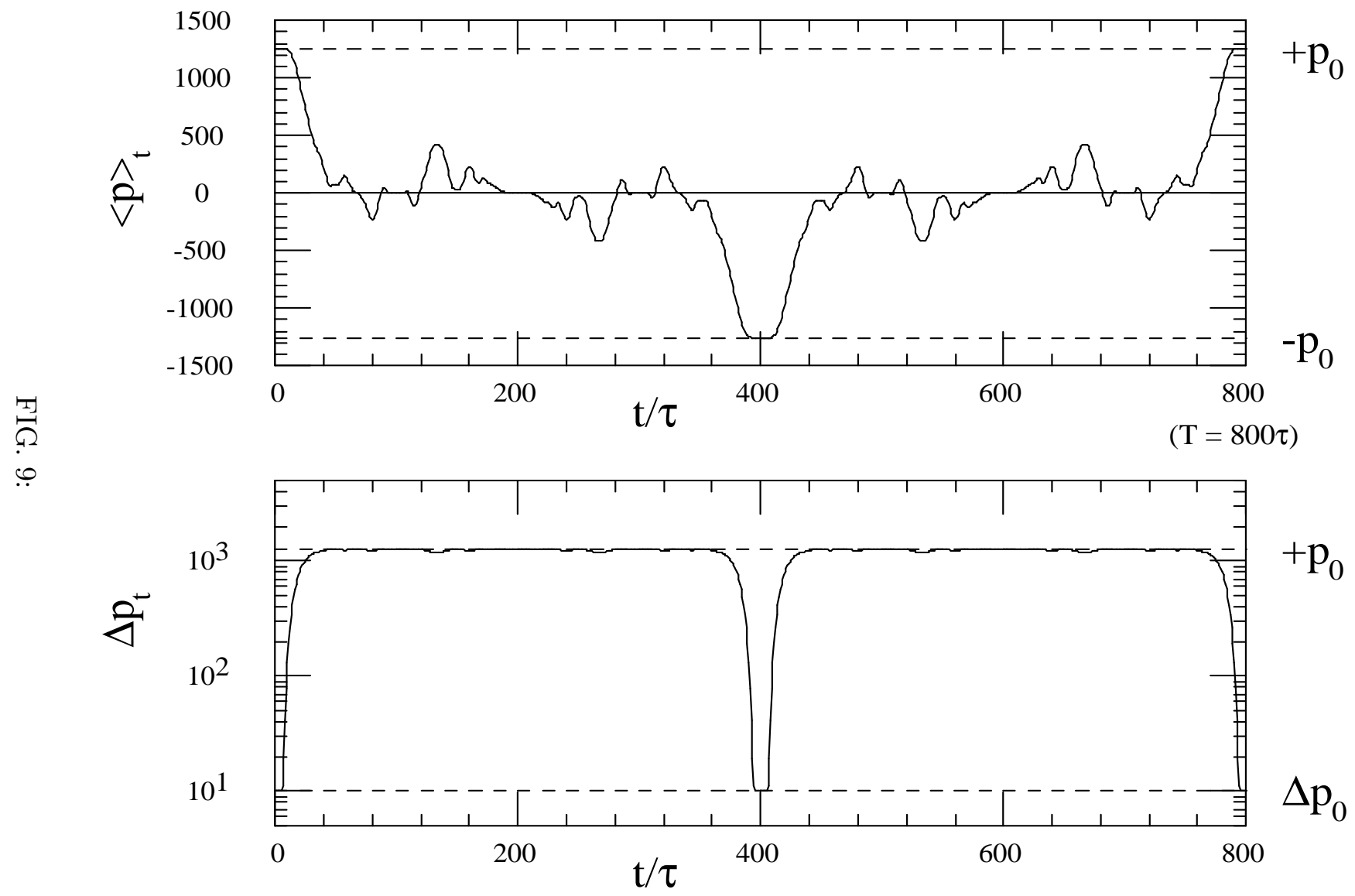




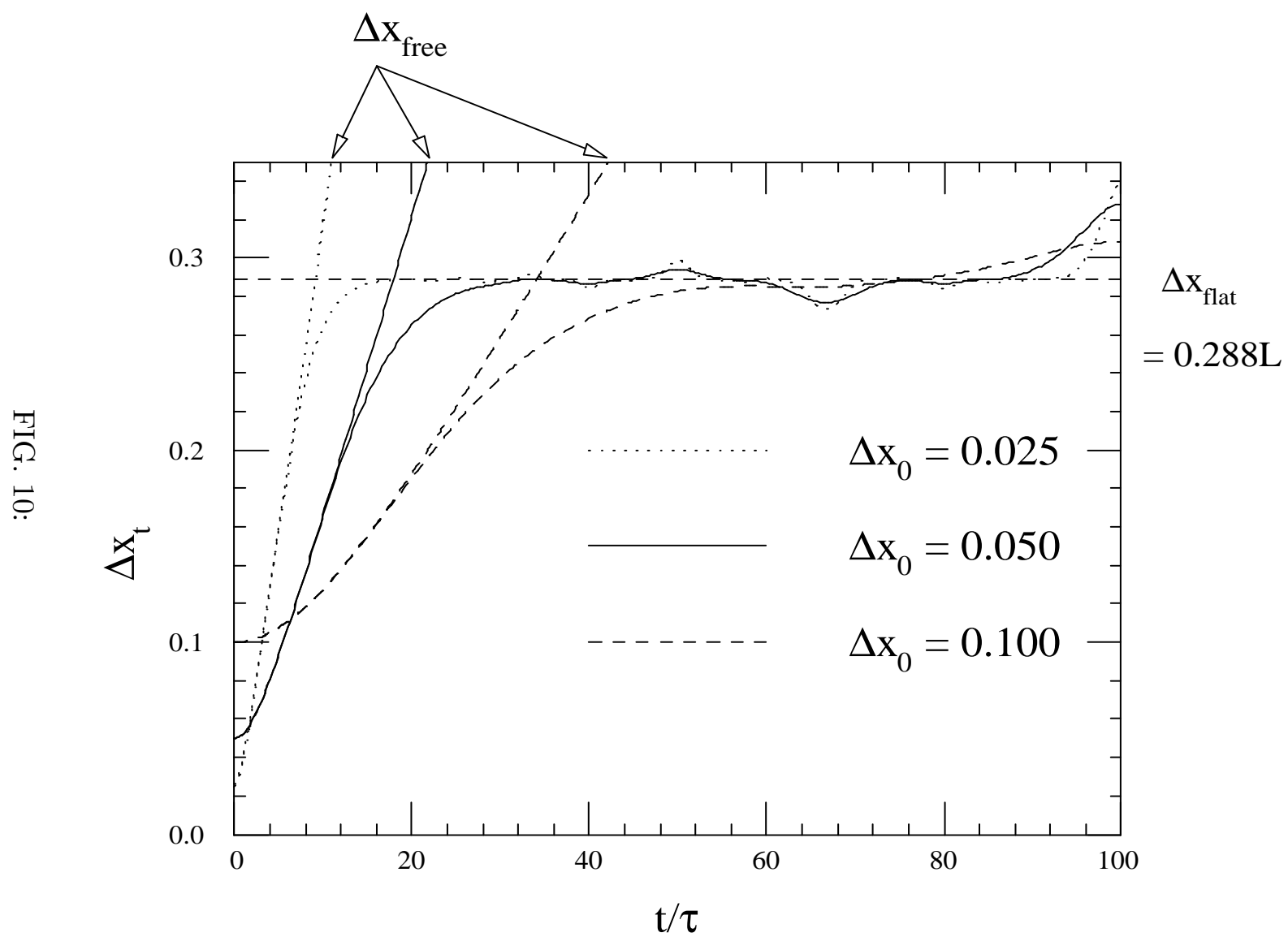



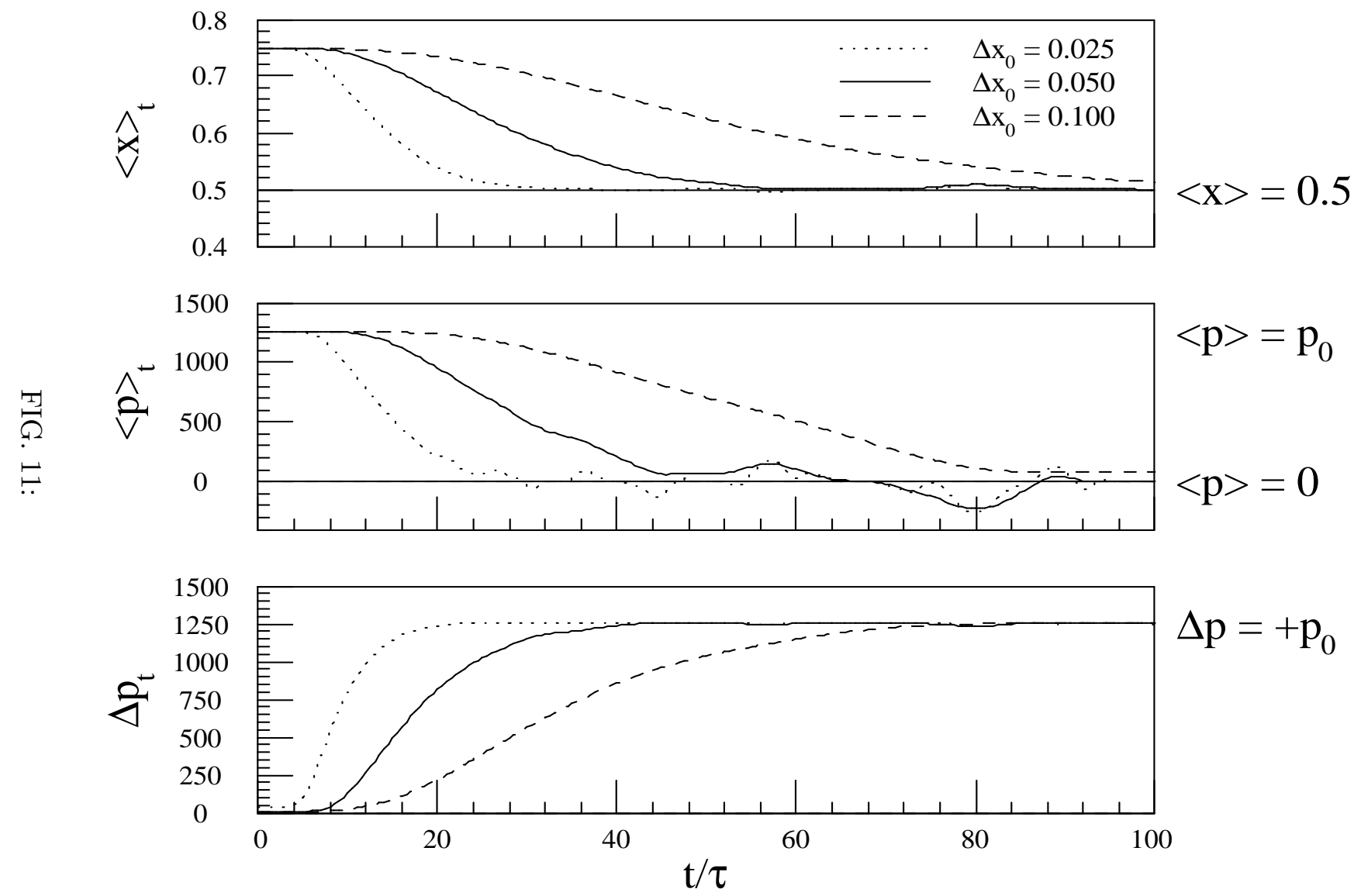